\title{
Restricted Kinematic Alignment: The Ideal Compromise?
}

\author{
William G. Blakeney and Pascal-André Vendittoli
}

\section{Key Points}

- The normal knee anatomy varies widely, and the more extreme ones may be inherently biomechanically inferior which may have deleterious effects on the TKA biomechanics and wear patterns.

- The restricted kinematic alignment protocol (rKA) has been developed as an alternative solution to the "true" KA technique in situations of patients with atypical knee anatomy.

- The rKA protocol limits the femoral and tibial prosthesis coronal alignment to within $\pm 5^{\circ}$ of neutral, with the overall combined lower limb coronal orientation within $\pm 3^{\circ}$ of neutral.

- $50 \%$ of patients fit within the rKA safe range allowing a pure KA technique. Minimal cor-

Electronic Supplementary Material The online version of this chapter (https://doi.org/10.1007/978-3-030-24243$5 \_17$ ) contains supplementary material, which is available to authorized users.

W. G. Blakeney

Department of Surgery, CIUSSS-de-L'Est-de-L'Ilede-Montréal, Hôpital Maisonneuve Rosemont,

Montréal, QC, Canada

Albany Health Campus, Albany, WA, Australia

P.-A. Vendittoli $(\bowtie)$

Department of Surgery, CIUSSS-de-L'Est-de-L'Ilede-Montréal, Hôpital Maisonneuve Rosemont, Montréal, QC, Canada

Department of Surgery, Université de Montréal, Montréal, QC, Canada rections are needed for one third, and more important anatomy changes are needed for the rest $(1 / 6)$.

- rKA protocol offers a satisfactory compromise, avoiding the important anatomy modifications and ligamentous releases required with MA but preventing the extremes of implant positioning that true KA technique may produce.

\subsection{Mechanical Alignment: The End of an Era}

Most patients following conventional total knee arthroplasty (TKA) do not experience a natural joint [1]. One fifth of patients are dissatisfied [2], over half may have residual symptoms [3], and up to a quarter would, in retrospect, not undergo the same surgery again [4]. Gait analysis studies have demonstrated that patients following TKA walked with less total range of knee motion and significant kinematic discrepancies [5].

When TKA was first introduced, instrument precision was poor, and implantation errors were frequent. There were many pitfalls to overcome; hence the focus was on implant survivorship, rather than reproducing normal knee function [6]. To achieve this, surgeons introduced the mechanical alignment (MA) technique. By selecting a neutral femoral and tibial cut with femoral rotation adapted and ligamentous releases to create equal femoral and extension gaps, a simpler 
method for alignment was created. This "one size fits all" approach, whilst reproducible, does not respect the wide range of normal anatomy of the knee [7]. Although the mean hip-knee-ankle angle (HKA) of patients scheduled for TKA is near neutral, a study of 4884 patients found only $0.1 \%$ of patients had both medial proximal tibial angle (MPTA) and lateral distal femoral angle (LDFA) at neutral, which is what MA aims to generate. Furthermore, a study on 1000 knee CT scans found that a systematic use of MA for TKA leads to many cases with gap asymmetries $[8,9]$. Mediolateral imbalances $>3 \mathrm{~mm}$ were created in $25 \%$ of varus and $54 \%$ of valgus knees. Using the trans-epicondylar axis for femoral rotation, only $49 \%$ of varus and $18 \%$ of valgus knees had $<3 \mathrm{~mm}$ of imbalance in both mediolateral and flexion-extension gaps. Some imbalances may not be correctable surgically and may explain residual TKA instability and poor results.

With a better understanding of normal knee joint anatomy and function, kinematic alignment (KA) technique has been introduced to improve clinical results following TKA. The KA technique for TKA aims to restore the pre-arthritic patient's constitutional lower limb alignment and joint surface orientations. It is a joint resurfacing procedure with only exceptional soft tissue release [10, 11]. We believe it is the end of the MA Era [12].

\subsection{Are All Anatomies Physiologic?}

The normal knee anatomy varies widely, and pathological changes increase its variability further [7, 13, 14]. In 4884 knees scheduled for TKA, HKA was $>3^{\circ}$ in $40 \%,>5^{\circ}$ in $19 \%$, and $>10^{\circ}$ in $3 \%$ of them [7]. The MPTA range was from $20.5^{\circ}$ varus to $20.5^{\circ}$ valgus, with a mean of $2.9^{\circ}$ varus. The LDFA range was from $11^{\circ}$ varus to $15.5^{\circ}$ valgus with a mean of $2.7^{\circ}$ valgus. The wide ranges demonstrate the huge variability in patient's anatomy (Fig. 17.1).

The more extreme anatomies may be inherently biomechanically inferior and may have been altered by factors that might predispose to degenerative disease such as trauma, tumours,
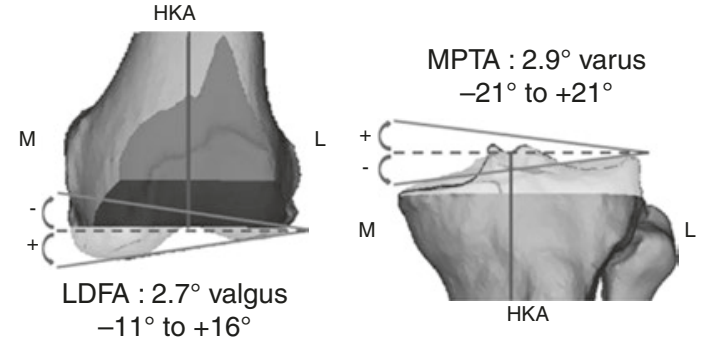

Fig. 17.1 Anatomic modification linked to mechanical alignment technique on the distal femur and proximal tibia

childhood deformity, or previous surgery (Fig. 17.2). A strong argument for the existence of patho-anatomies is the unilateral occurrence in some patients. We believe that surgeons should not blindly reproduce the same anatomy in these outlier patients as it may have deleterious effects on the TKA biomechanics and wear patterns. On the other hand, creating a neutral mechanical axis in these patients would be a significant anatomic modification and likely cause adjustments in soft tissue balance, differences in joint line orientation, variation of the femoral flexion axis, and alteration of knee kinematics.

A computer simulation study looking at the effects of MA or KA in TKA on a single knee model found that KA TKA produced nearnormal knee kinematics (with greater femoral rollback and more external rotation of the femoral component) [15]. However, there were also increased contact stresses, raising concerns about long-term outcomes. A retrieval study of 178 MA TKA revisions found that knees with greater varus alignment had increased total damage on the retrieved polyethylene inserts [16]. They also found that these MA TKAs tended to drift back towards the preoperative varus deformity before revision surgery, away from a neutral mechanical alignment. Other clinical [17] and simulator $[18,19]$ studies have similarly found an association between polyethylene wear and varus alignment. Greater tibial varus has also demonstrated a weak correlation $\left(r^{2}=0.45\right)$ with tibial baseplate migration at 10-year follow-up, in an RSA study [20]. Interestingly, overall limb alignment, from an HKA of $1.3^{\circ}$ valgus to over $10^{\circ}$ varus, did not affect baseplate migration. There was 


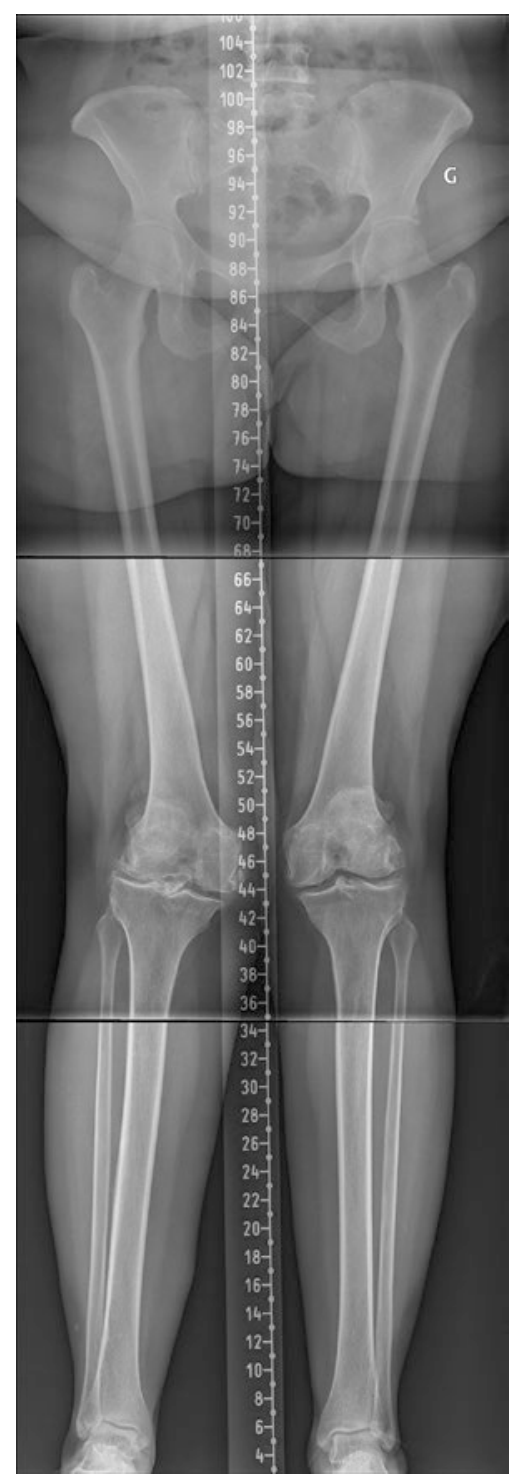

Fig. 17.2 Lower limb full-length radiographs show bilateral valgus lower limbs with severe right knee OA with LDFA of $11^{\circ}$ and MPTA of $6^{\circ}$. Reproducing her right lower limb alignment with systematic KA would leave her lower limb HKA in $5^{\circ}$ of valgus. We consider her right lower limb anatomy to be pathologic. One argument in favour of this assumption is the difference between the two lower limbs, with the left side being less extreme. Applying our protocol, we would reduce the LDFA to $5^{\circ}$ valgus and the MPTA to $2^{\circ}$ varus, resulting in a combined HKA of $3^{\circ}$ valgus

no difference between those within $\pm 3^{\circ}$ of neutral and those $>3^{\circ}$. These studies would suggest that systematically reproducing patients' patho- anatomy might not be suitable for implant survivorship using current TKA materials and fixation methods.

\subsection{Restricted Kinematic Alignment Protocol (rKA)}

The restricted kinematic alignment protocol (rKA) has been developed as an alternative solution to the "true" KA technique [11] in situations of patients with atypical knee anatomy. The concept of rKA is to reproduce the patient's constitutional knee anatomy within a safe range, avoiding the extreme pathological anatomies that have been demonstrated to exist [7]. The rKA protocol limits the femoral and tibial prosthesis coronal alignment to within $\pm 5^{\circ}$ of neutral, with the overall combined lower limb coronal orientation within $\pm 3^{\circ}$ of neutral (e.g. placing a femur in $4^{\circ}$ of valgus with a tibia in $5^{\circ}$ of varus would result in an overall combined coronal orientation of $1^{\circ}$ varus). To resurface the posterior condyles, a posterior referencing guide is set to neutral rotation, thus resecting only the implant thickness of the posterior condyles and matching each patient's native femoral orientation. Tibial baseplate rotation is set relative to the trial femoral component with the knee in extension.

The surgeon aims to reproduce the patient's normal anatomy as in the KA technique. This can be achieved using measured resection techniques with a calliper, intraoperative computer navigation or preoperative planning with patient-specific instrumentation. Resections are only modified from the patient's anatomy if the measured angles fall outside the predefined safe range. A study assessed the preoperative CT scans of 4884 knees undergoing TKA surgery to analyse the effect of the rKA technique [7]. This demonstrated that $51 \%$ of patients fell within the safe range allowing a pure KA technique. Allowing for minimal corrections (mean of $0.5^{\circ}$ for the tibia and $0.3^{\circ}$ for the femur), this was increased to $83 \%$ of all patients.

The protocol for performing these minimal corrections is as follows (Fig. 17.3). First, the surgeon 


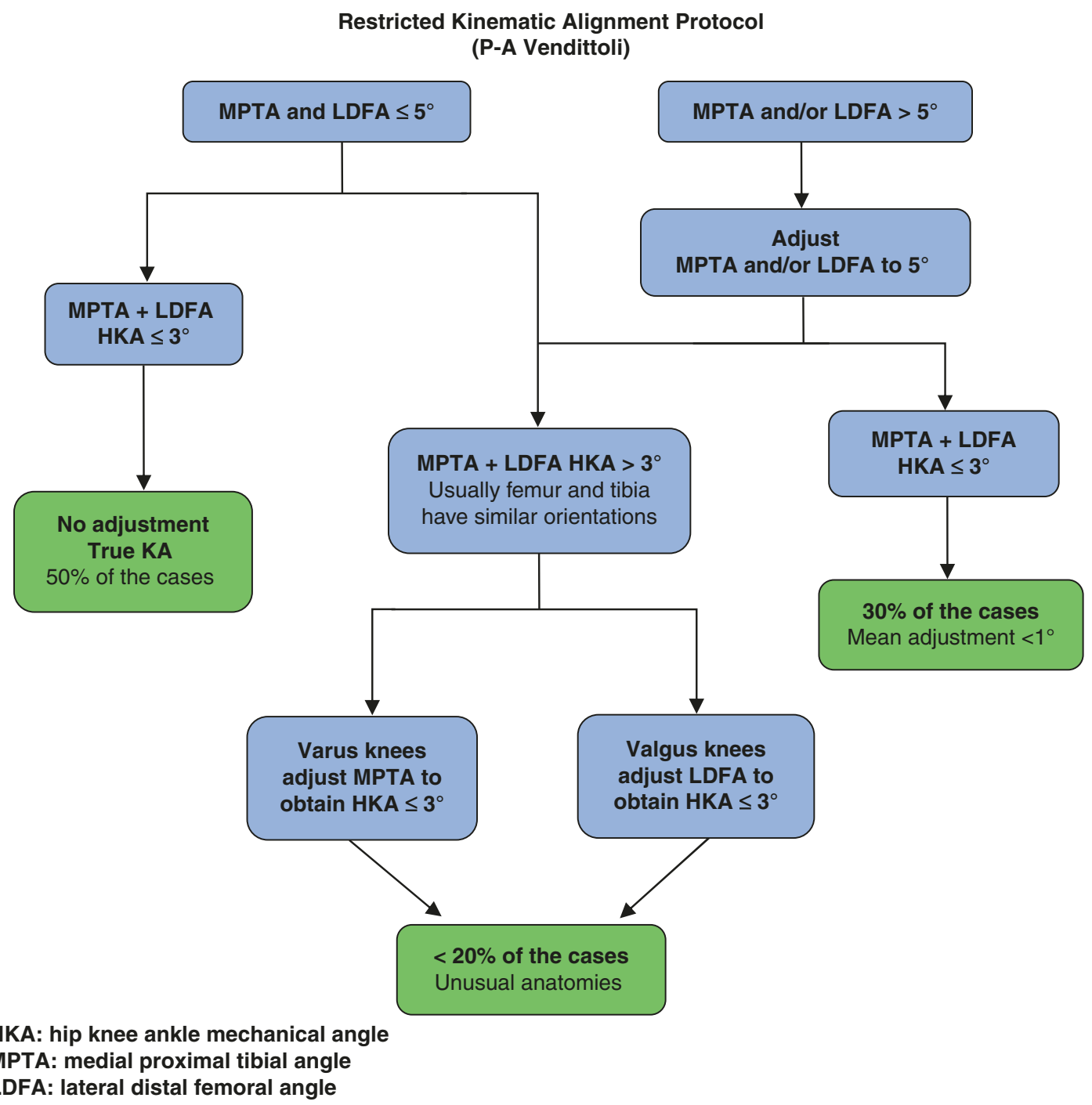

Fig. 17.3 rKA decision-making flow diagram

corrects the tibial and/or femoral bone cuts to fall within the $5^{\circ}$ limit. This will then correct the overall HKA to within $\pm 3^{\circ}$ of neutral in a significant proportion $(51 \%)$. In $8 \%$ of the cases, the patient maintained an HKA of $>3^{\circ}$ of varus (e.g. femur $1^{\circ}$ valgus and tibia $5^{\circ}$ varus $=\mathrm{HKA} 4^{\circ}$ varus). In these cases, the tibial varus was further reduced until the HKA was $3^{\circ}$ varus. In $7 \%$ of cases, the patient maintained an HKA of $>3^{\circ}$ of valgus (e.g. femur $5^{\circ}$ valgus and tibia $1^{\circ}$ varus $=$ HKA $4^{\circ}$ valgus). In these cases, the tibial varus was increased until the HKA was $3^{\circ}$ valgus. When anatomic correction is needed, we prefer to modify the tibia to preserve as much as possible the femoral anatomy and its flexion axis. Ligamentous releases are usually not needed in cases with anatomic modifications of $<3^{\circ}$. In larger corrections, minimal releases can be added (usually, to a much lesser degree compared to MA).

In our simulation study [7], 17\% of knees had unusual anatomy, with both the femur and tibia articular orientations being in varus or valgus. As both bones contribute the same direction to the overall HKA deviation, the surgeon needs to decide which bone to correct to fall into the safe range. As stated earlier, we believe that the femoral flexion axis plays the more significant role in knee kinematics, our practice is to pre- 
serve femoral anatomy as closely as possible and perform greater modifications on the tibial side. For example, in a valgus knee with a femur in $9^{\circ}$ valgus and a tibia with $1^{\circ}$ valgus (overall HKA of $10^{\circ}$ valgus), the femoral cut is modified to our maximum of $5^{\circ}$ valgus and the tibial cut corrected to $2^{\circ}$ varus, giving an overall HKA of $3^{\circ}$ valgus. Similarly, in a severe varus knee with $2^{\circ}$ varus femur and $6^{\circ}$ varus tibia (overall HKA of $8^{\circ}$ varus), the femoral orientation is maintained $\left(2^{\circ}\right.$ varus), and the tibial varus is reduced to $1^{\circ}$, giving an overall HKA of $3^{\circ}$ varus. One must consider that most of these cases have associated extraarticular deformities explaining these extremes HKAs. The severe valgus often has a tibia valga deformity in the diaphysis, and the severe varus may have a femoral bowing contributing to the lower limb alignment [21]. In these cases, resurfacing the knee joint (KA) will favour ligament laxities preservation but will not address the lower limb deviation linked to the extra-articular deformity. On the other hand, performing the rKA protocol will correct the extra-articular deformity with intra-articular cuts and may require ligament release/adjustment to avoid secondary instability.

The rKA protocol brings back the extreme anatomies towards acceptable values, modifying their deformities to allow an implant orientation compatible with current materials and fixation methods. On the other hand, simulating an MA technique in this same cohort of patients, significantly larger corrections were necessary [7]. The mean MPTA correction was $3.3^{\circ}$ for MA versus $0.5^{\circ}$ for $\mathrm{rKA}(p<0.001)$. Similarly, the mean LDFA correction was $3.2^{\circ}$ for MA versus $0.3^{\circ}$ for rKA $(p<0.001)$. This highlights that across a large population, performing MA requires significant changes to normal anatomy. These greater anatomic modifications then necessitate larger soft tissue releases to balance the knee, which may have detrimental effects on normal biomechanics.

\section{4 rKA Clinical Results}

A clinical series of the first 100 patients operated on using the rKA protocol demonstrated satisfactory functional outcomes at early follow-up (mean 2.4 years, range $1-3.7$ ). Only $5 \%$ of the knees required minor ligamentous release. A study of gait analysis comparing patients operated on with rKA compared to MA technique demonstrated that the rKA patients had knee kinematics that were significantly closer to healthy controls than MA patients [22]. The MA group displayed several significant knee kinematic differences to the healthy group: less sagittal plane range of motion $\left(49^{\circ}\right.$ vs. $\left.54^{\circ}, p=0.020\right)$, decreased maximum flexion $\left(52^{\circ}\right.$ vs. $\left.58^{\circ}, p=0.002\right)$, and increased adduction angle $\left(2.0-7.5^{\circ}\right.$ vs. $-2.8-3.0^{\circ}$, $p<0.05)$. These kinematic differences translated in significantly higher postoperative KOOS score in the KA group compared to the MA group (74 vs. $61, p=0.034)$.

In a study of 1000 preoperative CT scans of patients undergoing TKA, bone cuts, we compared the mediolateral and flexion-extension gap asymmetry between measured resection MA and rKA protocol bone cut simulations. Two MA techniques were simulated for rotation: using the surgical transepicondyar axis (TEA) and $3^{\circ}$ to the posterior condyles (PC). Extension space mediolateral (ML) imbalances ( $>2 \mathrm{~mm}$ ) occurred in $33 \%$ of TKA with MA technique versus $8 \%$ of the knees with rKA; imbalances ( $>4 \mathrm{~mm}$ ) were present in up to $11 \%$ of MA knees versus $1 \%$ rKA $(p<0.001)$. Using the MA technique, for the flexion space, higher ML imbalance rates were created by the TEA technique $(p<0.001)$. rKA again performed better than both MA techniques using TEA or $3^{\circ}$ PC techniques $(p<0.001)$. Using MA with TEA or PC, there were only $49 \%$ and $63 \%$ of the knees respectively with $<3 \mathrm{~mm}$ of imbalance throughout the extension and flexion spaces and medial and lateral compartments versus $92 \%$ using rKA $(p<0.001)$. Other studies have similarly reported that the MA technique frequently results in significant anatomical modifications with a wide range of complex collateral ligament imbalances, which are not correctable by collateral ligament release [7, 23].

\section{5 rKA Versus True KA: A Compromise?}

Many surgeons worry about leaving too much varus or valgus with the KA technique. Howell 
et al. [24] observed $97.5 \%$ implant survivorship in a cohort of $208 \mathrm{KA}$ TKAs at 6-year followup, with no increased failure in those with greater varus. In another study, radiostereometric analysis of TKAs randomised to MA or KA did not discern significant differences in implant migration between groups [25]. There are no long-term follow-up studies on KA TKAs, whereas MA TKAs have a long history of good survivorship [26-28]. Achieving a mechanical axis within $3^{\circ}$ of neutral has been associated with better functional outcomes than malaligned TKAs in some studies [29-31]. Other studies have demonstrated increased aseptic loosening and rates of failure with malaligned components [32-34]. In contrast, more recent studies have failed to demonstrate greater survivorship or functional outcomes in well-aligned prostheses (within $\pm 3^{\circ}$ of neutral) compared to malaligned TKAs [35-38]. The results of these studies should be generalised to KA with caution. It must be understood that accurate KA, aiming for an HKA other than neutral, is very different from a malaligned TKA when aiming for neutral. There are no doubt other factors than coronal prosthesis alignment that affect how the knee will be loaded dynamically. Studies, both in asymptomatic patients [39] and in kinematic TKA patients [40], have demonstrated that despite a range in alignment, the joint line remains parallel to the ground when standing. The resultant functional joint line orientation may well be favourable for the overall load profile of the prosthetic joint.

In the absence of further evidence from long-term studies of KA TKAs, however, some authors have cautioned against widespread adoption of KA technique [41]. We believe the rKA protocol offers a satisfactory compromise, allowing recreation of normal patient anatomy for the majority of cases, avoiding the excessive corrections and ligamentous releases required with MA, but preventing the extremes of implant positioning that an unrestricted KA technique may produce.

\subsection{Case Example}

\subsubsection{Case Example 1}

A 65-year-old male, with severe varus right knee OA. Preoperative long leg radiographs (see Fig. 17.4) demonstrate right femoral varus (LDFA of $93^{\circ}$ ) with a valgus tibia (MPTA of $88^{\circ}$ ). The patient elected to undergo right TKA using our rKA protocol. In this case, as is the case with $\sim 50 \%$ of cases, no modifications were required from his preoperative constitutional alignment allowing a pure KA approach. Although his resultant HKA is near neutral ( $1^{\circ}$ varus), his joint surface orientations were maintained. With MA technique, both the femoral and tibial anatomies would have been significantly modified to neutral. This joint line orientation and flexion axis changes would affect knee kinematics. It is interesting to note that in such a case, using intramedullary rod alignment for the tibia would lead to an important error in valgus.

\subsubsection{Case Example 2}

An active 58-year-old female, with painful right knee OA and previous left knee MA TKA. Preoperative planning long radiographs demonstrate right femoral valgus (LDFA of $83^{\circ}$ ) with a neutral tibia (MPTA of $90^{\circ}$ ) (see Fig. 17.5). The patient elected to undergo right TKA using our rKA protocol. In this case, to stay within our rKA safe range, we increased her LDFA to $85^{\circ}$ (reducing valgus from $7^{\circ}$ to $5^{\circ}$ ) and reduced her MPTA to $88^{\circ}$ (from neutral to $2^{\circ}$ varus). Resultant HKA was valgus $3^{\circ}$. With such corrections, we tried to minimise femoral axis and femoral anatomy modifications. No ligament release was needed. The patient underwent an uneventful postoperative recovery. At 4 months post-surgery, she feels she has a right forgotten knee without restrictions. On the left MA TKA, even with a LDFA and MPTA at $90^{\circ}$, the resulting HKA is $3^{\circ}$ varus. The patient prefers her right TKA. 
Fig. 17.4 Standing long radiographs of case example 1

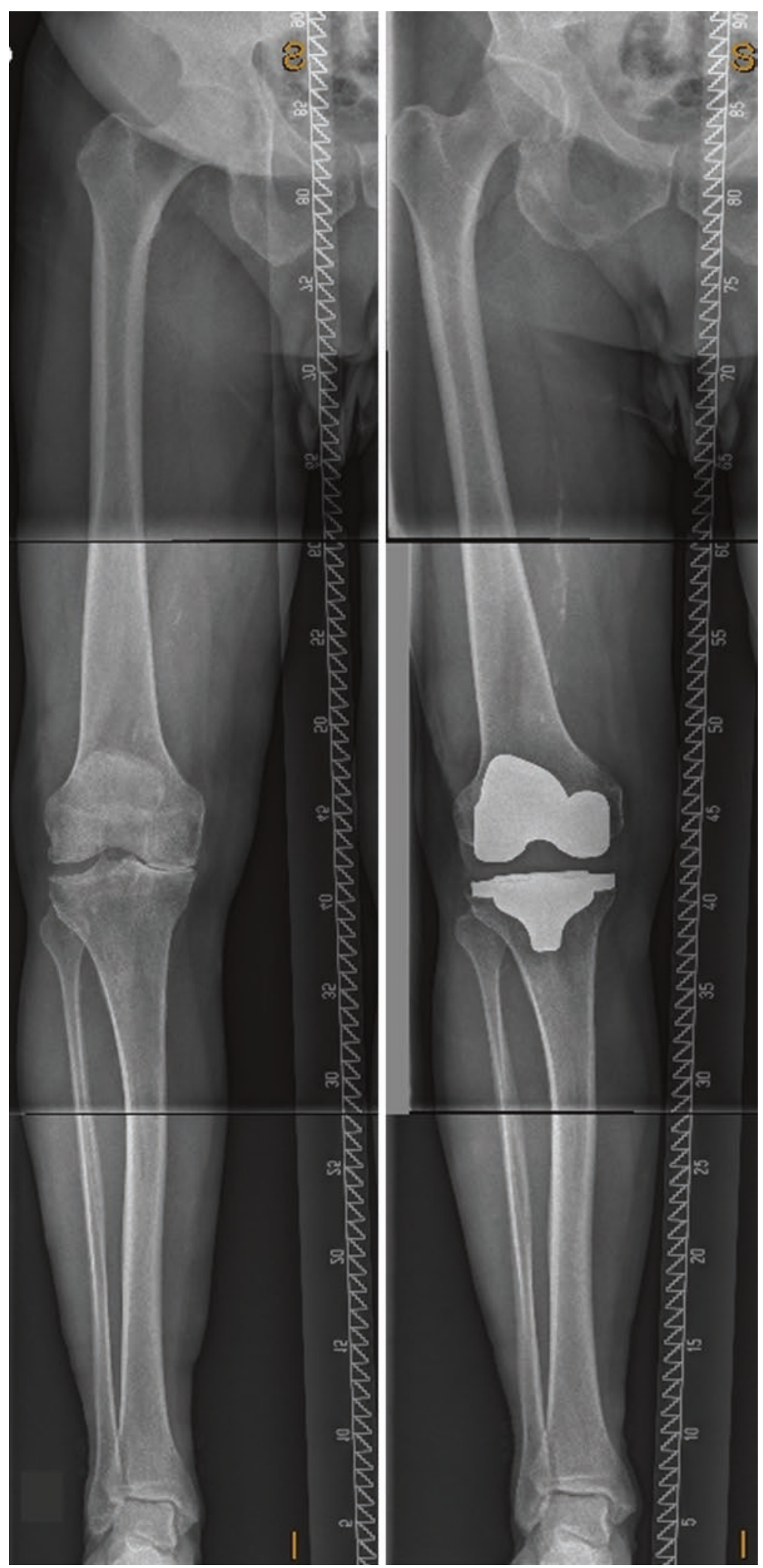



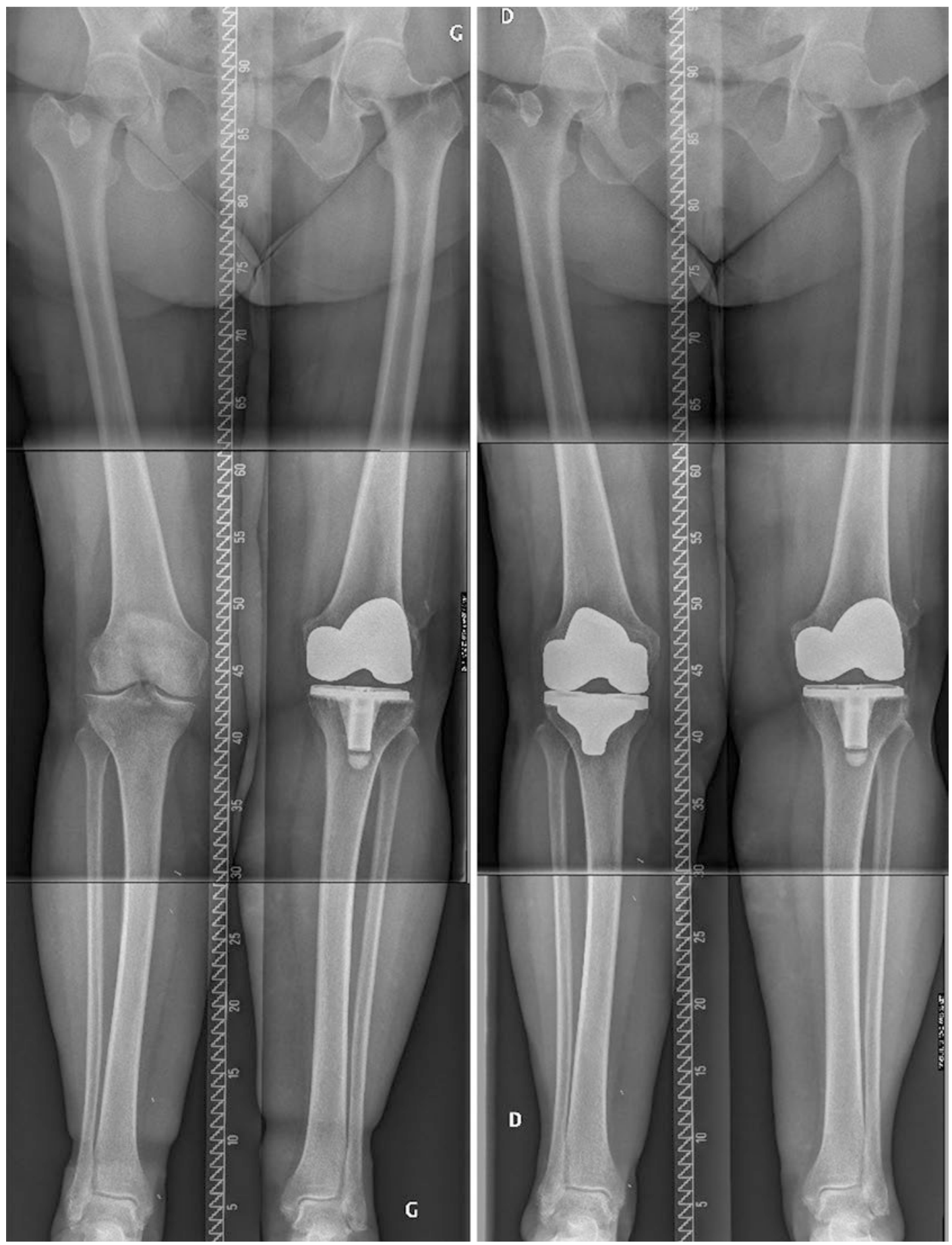

Fig. 17.5 Standing long radiographs of case example number 2 


\section{References}

1. Collins M, Lavigne M, Girard J, Vendittoli PA. Joint perception after hip or knee replacement surgery. Orthop Traumatol Surg Res. 2012;98:275-80.

2. Bourne RB, Chesworth BM, Davis AM, Mahomed NN, Charron KDJ. Patient satisfaction after total knee arthroplasty: who is satisfied and who is not? Clin Orthop Relat Res. 2010;468:57-63.

3. Nam D, Nunley RM, Barrack RL. Patient dissatisfaction following total knee replacement: a growing concern? Bone Joint J. 2014;96-B:96-100.

4. Lingard EA, Sledge CB, Learmonth ID, Kinemax Outcomes G. Patient expectations regarding total knee arthroplasty: differences among the United States, United Kingdom, and Australia. J Bone Joint Surg Am. 2006;88:1201-7.

5. McClelland JA, Webster KE, Feller JA. Gait analysis of patients following total knee replacement: a systematic review. Knee. 2007;14:253-63.

6. Vendittoli PA, Blakeney W. Redefining knee replacement. Orthop Traumatol Surg Res. 2017;103:977-9.

7. Almaawi AM, Hutt JRB, Masse V, Lavigne M, Vendittoli P-A. The impact of mechanical and restricted kinematic alignment on knee anatomy in total knee arthroplasty. J Arthroplast. 2017;32:2133-40.

8. Blakeney W, Beaulieu Y, Puliero B, Kiss MO, Vendittoli PA. Bone resection for mechanically aligned total knee arthroplasty creates frequent gap modifications and imbalances. Knee Surg Sports Traumatol Arthrosc. 2019. https://doi.org/10.1007/s00167-019-05562-8.

9. Blakeney W, Beaulieu Y, Kiss MO, Rivière C, Vendittoli PA. Less gap imbalance with restricted kinematic alignment than with mechanically aligned total knee arthroplasty: simulations on 3-D bone models created from CT-scans. Acta Orthop. 2019;90(6):602-9.

10. Howell SM, Howell SJ, Hull ML. Assessment of the radii of the medial and lateral femoral condyles in varus and valgus knees with osteoarthritis. J Bone Joint Surg Am. 2010;92:98-104.

11. Hutt JRB, LeBlanc MA, Massé V, Lavigne M, Vendittoli PA. Kinematic TKA using navigation: surgical technique and initial results. Orthop Traumatol Surg Res. 2016;102:99-104.

12. Rivière C, Vigdorchik JM, Vendittoli PA. Mechanical alignment: The end of an era! Orthop Traumatol Surg Res. 2019;105(7):1223-6. https://doi.org/10.1016/j. otsr.2019.07.005

13. Bellemans J, Colyn W, Vandenneucker H, Victor J. The Chitranjan Ranawat award: is neutral mechanical alignment normal for all patients? The concept of constitutional varus. Clin Orthop Relat Res. 2012;470:45-53.

14. Eckhoff DG, Bach JM, Spitzer VM, et al. Threedimensional mechanics, kinematics, and morphology of the knee viewed in virtual reality. J Bone Joint Surg Am. 2005;87(Suppl 2):71-80.
15. Ishikawa M, Kuriyama S, Ito H, Furu M, Nakamura S, Matsuda S. Kinematic alignment produces nearnormal knee motion but increases contact stress after total knee arthroplasty: a case study on a single implant design. Knee. 2015;22:206-12.

16. Li Z, Esposito CI, Koch CN, Lee YY, Padgett DE, Wright TM. Polyethylene damage increases with varus implant alignment in posterior-stabilized and constrained condylar knee arthroplasty. Clin Orthop Relat Res. 2017;475:2981-91.

17. Srivastava A, Lee GY, Steklov N, Colwell CW Jr, Ezzet KA, D'Lima DD. Effect of tibial component varus on wear in total knee arthroplasty. Knee. 2012;19:560-3.

18. Werner FW, Ayers DC, Maletsky LP, Rullkoetter PJ. The effect of valgus/varus malalignment on load distribution in total knee replacements. J Biomech. 2005;38:349-55.

19. D'Lima DD, Hermida JC, Chen PC, Colwell CW. Polyethylene wear and variations in knee kinematics. Clin Orthop Relat Res. 2001;392:124-30.

20. Teeter MG, Naudie DD, McCalden RW, et al. Varus tibial alignment is associated with greater tibial baseplate migration at 10 years following total knee arthroplasty. Knee Surg Sports Traumatol Arthrosc. 2018;26:1610-7.

21. Alghamdi A, Rahme M, Lavigne M, Masse V, Vendittoli PA. Tibia valga morphology in osteoarthritic knees: importance of preoperative full limb radiographs in total knee arthroplasty. J Arthroplast. 2014;29:1671-6.

22. Blakeney W, Clément J, Desmeules F, Hagemeister $\mathrm{N}$, Rivière $\mathrm{C}$, Vendittoli PA. Kinematic alignment in total knee arthroplasty better reproduces normal gait than mechanical alignment. Knee Surg Sports Traumatol Arthrosc. 2019;27(5):1410-7. https://doi.org/10.1007/s00167-018-5174-1.

23. Gu Y, Roth JD, Howell SM, Hull ML. How frequently do four methods for mechanically aligning a total knee arthroplasty cause collateral ligament imbalance and change alignment from normal in white patients? AAOS exhibit selection. J Bone Joint Surg Am. 2014;96:e101.

24. Howell SM, Papadopoulos S, Kuznik K, Ghaly LR, Hull ML. Does varus alignment adversely affect implant survival and function six years after kinematically aligned total knee arthroplasty? Int Orthop. 2015;39:2117-24.

25. Laende E, Richardson G, Biddulph M, Dunbar M. Implant fixation and gait analysis at one year following total knee arthroplasty with patient specific cutting blocks versus computer navigation. Bone Jt J. 2016;98-B:136.

26. Font-Rodriguez DE, Scuderi GR, Insall JN. Survivorship of cemented total knee arthroplasty. Clin Orthop Relat Res. 1997;345:79-86.

27. Gill GS, Joshi AB, Mills DM. Total condylar knee arthroplasty. 16- to 21-year results. Clin Orthop Relat Res. 1999;367:210-5. 
28. Rodricks DJ, Patil S, Pulido P, Colwell CW. Pressfit condylar design total knee arthroplasty. Fourteen to seventeen-year follow-up. J Bone Joint Surg Am. 2007;89:89-95.

29. Choong PF, Dowsey MM, Stoney JD. Does accurate anatomical alignment result in better function and quality of life? Comparing conventional and computer-assisted total knee arthroplasty. J Arthroplast. 2009;24:560-9.

30. Blakeney WG, Khan RJK, Palmer JL. Functional outcomes following total knee arthroplasty: a randomised trial comparing computer-assisted surgery with conventional techniques. Knee. 2014;21:364-8.

31. Longstaff LM, Sloan K, Stamp N, Scaddan M, Beaver R. Good alignment after total knee arthroplasty leads to faster rehabilitation and better function. J Arthroplast. 2009;24:570-8.

32. Berend ME, Ritter MA, Meding JB, et al. Tibial component failure mechanisms in total knee arthroplasty. Clin Orthop Relat Res. 2004;428:26-34.

33. Fang DM, Ritter MA, Davis KE. Coronal alignment in total knee arthroplasty: just how important is it? J Arthroplasty. 2009;24:39-43.

34. Jeffery RS, Morris RW, Denham RA. Coronal alignment after total knee replacement. J Bone Joint Surg Br. 1991;73:709-14.

35. Abdel MP, Ollivier M, Parratte S, Trousdale RT, Berry DJ, Pagnano MW. Effect of postoperative mechanical axis alignment on survival and functional outcomes of modern total knee arthroplasties with cement: a concise follow-up at 20 years. J Bone Joint Surg Am. 2018;100:472-8.

36. Parratte S, Pagnano MW, Trousdale RT, Berry DJ. Effect of postoperative mechanical axis alignment on the fifteen-year survival of modern, cemented total knee replacements. J Bone Joint Surg Am. 2010;92:2143-9.

37. Morgan SS, Bonshahi A, Pradhan N, Gregory A, Gambhir A, Porter ML. The influence of postoperative coronal alignment on revision surgery in total knee arthroplasty. Int Orthop. 2008;32:639-42.

38. Bonner TJ, Eardley WGP, Patterson P, Gregg PJ. The effect of post-operative mechanical axis alignment on the survival of primary total knee replacements after a follow-up of 15 years. J Bone Joint Surg Br. 2011;93:1217-22.

39. Victor JM, Bassens D, Bellemans J, Gursu S, Dhollander AA, Verdonk PC. Constitutional varus does not affect joint line orientation in the coronal plane. Clin Orthop Relat Res. 2014;472:98-104.

40. Hutt J, Massé V, Lavigne M, Vendittoli P-A. Functional joint line obliquity after kinematic total knee arthroplasty. Int Orthop. 2016;40:29-34.

41. Abdel MP, Oussedik S, Parratte S, Lustig S, Haddad FS. Coronal alignment in total knee replacement: historical review, contemporary analysis, and future direction. Bone Jt J. 2014;96-B:857-62.

Open Access This chapter is licensed under the terms of the Creative Commons Attribution 4.0 International License (http://creativecommons.org/licenses/by/4.0/), which permits use, sharing, adaptation, distribution and reproduction in any medium or format, as long as you give appropriate credit to the original author(s) and the source, provide a link to the Creative Commons license and indicate if changes were made.

The images or other third party material in this chapter are included in the chapter's Creative Commons license, unless indicated otherwise in a credit line to the material. If material is not included in the chapter's Creative Commons license and your intended use is not permitted by statutory regulation or exceeds the permitted use, you will need to obtain permission directly from the copyright holder. 